INPLASY

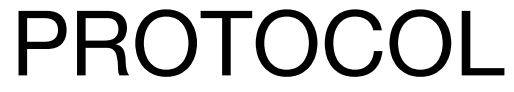

To cite: Wu et al. The efficacy of transcranial direct current stimulation in enhancing surgical skill acquisition. Inplasy protocol 202140099. doi:

10.37766/inplasy2021.4.0099

Received: 19 April 2021

Published: 19 April 2021

Corresponding author:

Ping-Tao Tseng

ducktseng@gmail.com

Author Affiliation:

Prospect Clinic for

Otorhinolaryngology \&

Neurology, Kaohsiung City,

Taiwan

Support: None.

Review Stage at time of this submission: Data analysis.

Conflicts of interest:

None declared.

\section{The efficacy of transcranial direct current stimulation in enhancing surgical skill acquisition}

\author{
Wu, MK¹; Tseng, PT².
}

Review question / Objective: The application of transcranial direct current stimulation (tDCS) to targeted cortices (often the primary motor cortex) has been found to be associated with significant improvements in skill acquisition. However, these beneficial effects have still been controversial.

Condition being studied: To investigate the association between the application of tDCS and the efficacy of surgical performance during surgical skill training.

Information sources: PubMed, Embase, ClinicalKey, Cochrane CENTRAL, ProQuest, ScienceDirect, Web of Science, and ClinicalTrials.gov platforms, contact with authors, trial registration site, and grey literature.

INPLASY registration number: This protocol was registered with the International Platform of Registered Systematic Review and Meta-Analysis Protocols (INPLASY) on 19 April 2021 and was last updated on 19 April 2021 (registration number INPLASY202140099).

\section{INTRODUCTION}

Review question / Objective: The application of transcranial direct current stimulation (tDCS) to targeted cortices (often the primary motor cortex) has been found to be associated with significant improvements in skill acquisition. However, these beneficial effects have still been controversial.

Rationale: Although many meta-analyses have demonstrated the benefit of tDCS in the improvement of gross motor activities, no previous meta-analysis has specifically investigated the benefit of tDCS in surgical skill acquisition, which was recognized to 
involve complex visuospatial memory, executive function, attention, and fine movement.

Condition being studied: To investigate the association between the application of tDCS and the efficacy of surgical performance during surgical skill training.

\section{METHODS}

Search strategy: PubMed, Embase, ClinicalKey, Cochrane CENTRAL, ProQuest, ScienceDirect, Web of Science, and ClinicalTrials.gov platforms.

Participant or population: Participants receiving surgical skill training.

Intervention: Transcranial direct-current stimulation.

Comparator: Sham-control.

Study designs to be included: Randomized controlled trials.

Eligibility criteria: (1) randomized controlled trials (RCTs) investigating the difference in changes of surgical performance after transcranial direct-current stimulation (tDCS) or sham stimulation, (2) RCTs of either sham-control or active-control, and (3) RCTs investigating different efficacies in surgical skill training with the tDCS intervention.

Information sources: PubMed, Embase, ClinicalKey, Cochrane CENTRAL, ProQuest, ScienceDirect, Web of Science, and ClinicalTrials.gov platforms, contact with authors, trial registration site, and grey literature

Main outcome(s): The primary outcome was the change in surgical performance associated with NIBS or sham stimulation.

Additional outcome(s): Secondary outcomes were the changes in error scores. The error scores were defined as improper transfers in laparoscopic training or resection of healthy brain in neurosurgical training. Safety profile was defined as the rate of local discomfort (i.e., itching, tingling pain, or erythematous).

Data management: To extract data and input into a predetermined list in the excel file.

Quality assessment / Risk of bias analysis: Cochrane risk-of-bias tool, which consisted of seven main items (randomization, concealment, blindness of participants, blindness of investigator, attrition bias, reporting bias, and other bias).

Strategy of data synthesis: The current meta-analysis was conducted with random-effects meta-analysis models. The meta-analysis procedure was performed with Comprehensive Meta-Analysis software, version 3.

Subgroup analysis: Subgroup metaanalysis according to the cortex at which the NIBS was targeted.

Sensitivity analysis: A sensitivity test was performed using the one study removal method, in which one study was excluded from the analyses at a time to observe whether significant or insignificant results of the meta-analyses were biased by outliers.

Language: No restriction.

Country(ies) involved: Taiwan.

Keywords: surgical skill; resident training; meta-analysis; tDCS; neuromodulation.

Dissemination plans: Peer-review publication.

Contributions of each author:

Author 1 - Ming-Kung Wu - contributed as corresponding author.

Email: mingkung180@gmail.com

Author 2 - Ping-Tao Tseng - contributed as corresponding author.

Email: ducktseng@gmail.com 